\title{
Trajectory of the anomalous Hall effect towards the quantized state in a ferromagnetic topological insulator
}

\author{
J. G. Checkelsky ${ }^{1 \star i}$, R. Yoshimii ${ }^{1}$ A. Tsukazaki ${ }^{2}$, K. S. Takahashi' ${ }^{3}$, Y. Kozuka ${ }^{1}$, J. Falson ${ }^{1}$, M. Kawasaki ${ }^{1,3}$ \\ and Y. Tokura ${ }^{1,3}$
}

Topological insulators are bulk electronic insulators with symmetry-protected gapless modes on their surfaces ${ }^{1-5}$. Breaking time-reversal symmetry in these systems via ferromagnetism induces a gapped state characterized by a zero magnetic field quantized Hall response-the quantum anomalous Hall (QAH) state $^{6-8}$. A key question that has remained experimentally unexplored is the relationship of this new type of quantum Hall (QH) state with the previously known orbitally driven QH states. Here, we show experimentally that a ferromagnetic topological insulator exhibiting the QAH state is well described by the global phase diagram of the $\mathrm{QH}$ effect. By mapping the conductivity tensor in the vicinity of the QAH phase, we find evidence for quantum criticality and delocalization behaviour9,10 that can quantitatively be described by the renormalization group properties of the QH ground state ${ }^{11,12}$. This result demonstrates that this QAH state can be understood within the context of the law of corresponding states that governs the QH state ${ }^{13,14}$.

The introduction of magnetism has proved to be an incisive method to experimentally access the exotic nature of topological insulators (TIs). Early theoretical work predicted ordering of the magnetic spins at the surface of TIs would open a gap at the Dirac point in the protected surface electronic spectrum ${ }^{15,16}$. This gap was observed by angle-resolved photoemission spectroscopy (ARPES) experiments in both bulk ${ }^{17}$ and surface ${ }^{18}$ magnetically doped TIs. Such bulk ${ }^{19}$ and surface ${ }^{20}$ magnetic orderings were also observed to interact with the electronic transport of the surface modes. The nature of the ground state within the electronic gap was predicted to be characterized by a zero-field quantized Hall response (the QAH effect) in electronic transport ${ }^{6}$ accessible under the condition of sufficient electronic localization ${ }^{7}$. This line of inquiry culminated in the experimental observation of the QAH state in ferromagnetic TIs at low temperature ${ }^{8}$. With this discovery, significant interest has been focused on understanding the nature of the QAH state and its relation to conventional quantum Hall $(\mathrm{QH})$ states.

Here we establish an understanding of these symmetry-broken TI surface modes within the general context of the stable ground states of two-dimensional (2D) systems. In the presence of disorder and absence of magnetic fields, it is known that as the temperature $T$ goes to zero that $2 \mathrm{D}$ systems do not support electrical conduction ${ }^{21}$. With the introduction of a magnetic field, there is an additional stable state characterized by vanishing longitudinal conductance and quantized Hall conductance $n e^{2} / h$ ( $n$ is a non-zero integer, $e$ is the electronic charge and $h$ is Planck's constant): the QH liquid ${ }^{22}$. $2 \mathrm{D}$ systems subject to time-reversal symmetry (TRS) breaking due to ferromagnetism rather than external magnetic fields have also been shown theoretically to support stable insulating and QH liquid states $^{23}$. We are thus led to consider the surface states of magnetically doped TIs as 2D transport systems in the presence of symmetrybreaking ferromagnetism. As we show below, in the presence of magnetic order these states can be successfully understood as stabilized QH liquids and are fully characterized by the universal delocalization response known in $\mathrm{QH}$ systems.

We have grown thin films of the topological insulator $\mathrm{Cr}_{x}\left(\mathrm{Bi}_{1-y} \mathrm{Sb}_{y}\right)_{2-x} \mathrm{Te}_{3}$ on semi-insulating $\operatorname{InP}(111)$ substrates using molecular beam epitaxy (Methods). The films presented here have a thickness $t$ of approximately $8 \mathrm{~nm}$, as estimated from the calibrated growth rate and a fixed $\mathrm{Bi} / \mathrm{Sb}$ ratio set by $y=0.8$. For this $y$ value, it has been shown by ARPES that the Dirac point of the surface states is isolated within the bulk electronic band gap ${ }^{24}$. To demonstrate the effect of the magnetic dopant $\mathrm{Cr}$, we compare results for the growth of pristine $(x=0)$ and doped $(x=0.22)$ films. Figure 1a,b shows X-ray diffraction patterns for the pristine and doped films, respectively; in each case all peaks can be identified with $\left(\begin{array}{lll}0 & 0 & 0\end{array}\right)$ diffraction of $\left(\mathrm{Bi}_{1-y} \mathrm{Sb}_{y}\right)_{2} \mathrm{Te}_{3}$ or the $\mathrm{InP}(n n n)$ peaks. Figure 1c shows a detailed view of the $\left(\begin{array}{llll}0 & 0 & 0 & 15\end{array}\right)$ peak, exhibiting Laue fringes consistent with our estimated $t$. A compression of the $c$-axis lattice parameter is apparent in the Cr-doped film, reducing from $3.047 \mathrm{~nm}$ to $3.026 \mathrm{~nm}$, suggestive of $\mathrm{Cr}$ replacing $\mathrm{Bi}$. The left and right insets of Fig. 1c show the topography of the pristine and doped films, respectively, taken by atomic force microscopy. The pristine film shows an almost atomically flat surface-that is, it is comprised almost entirely of two quintuple layer (QL) levels separated by the QL thickness $(1 \mathrm{~nm})$-whereas the surface of the doped film shows approximately three QL levels. From this comparison we conclude that the introduction of $\mathrm{Cr}$ increases disorder in the film, but the film nevertheless retains the expected crystalline structure and quality.

We next compare the electronic transport properties of the films. Figure $1 \mathrm{~d}$ shows the resistance $R$ as a function of temperature $T$ for both films. Decreasing from $T=300 \mathrm{~K}$, the pristine film exhibits a non-metallic $R(T)$ to $T \approx 80 \mathrm{~K}$, followed by metallic behaviour to $10 \mathrm{~K}$, and finally an upturn to $2 \mathrm{~K}$. As has been previously discussed, these regimes arise when the chemical potential $\mu$ resides

\footnotetext{
${ }^{1}$ Department of Applied Physics and QPEC, University of Tokyo, Hongo, Tokyo 113-8656, Japan, ${ }^{2}$ Institute for Materials Research, Tohoku University, Sendai 980-8577, Japan, ${ }^{3}$ RIKEN Center for Emergent Matter Science (CEMS), Wako 351-0198, Japan. †Present address: Department of Physics, Massachusetts Institute of Technology, Cambridge, Massachusetts 02139, USA. *e-mail: checkelsky@mit.edu
} 


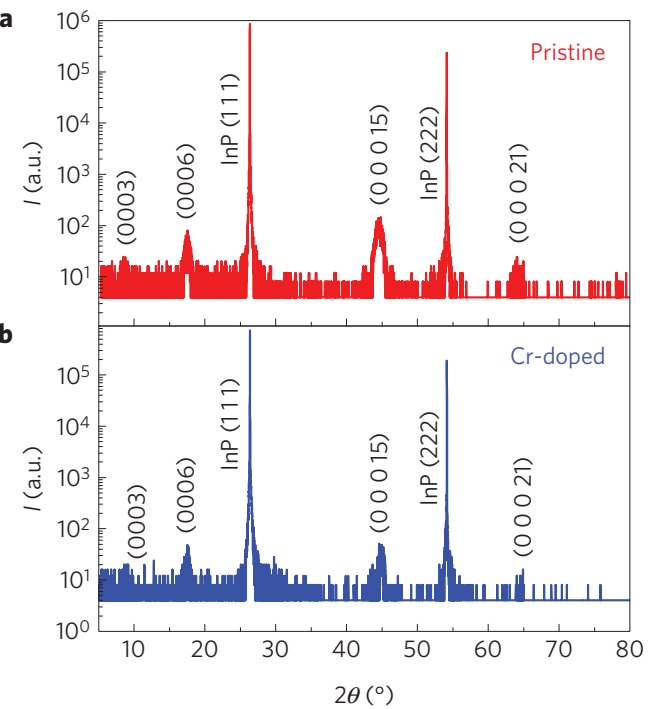

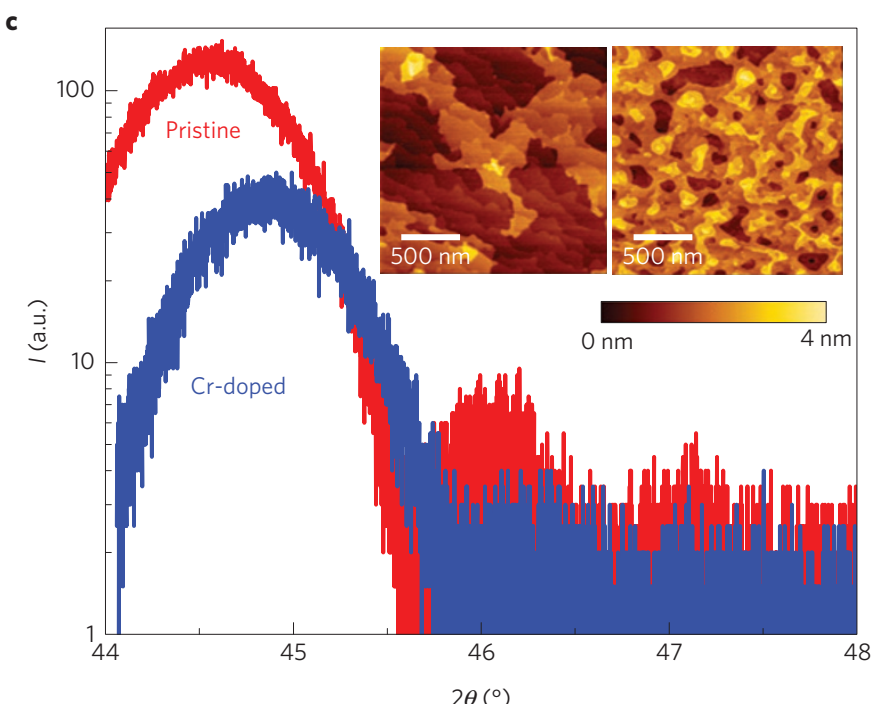

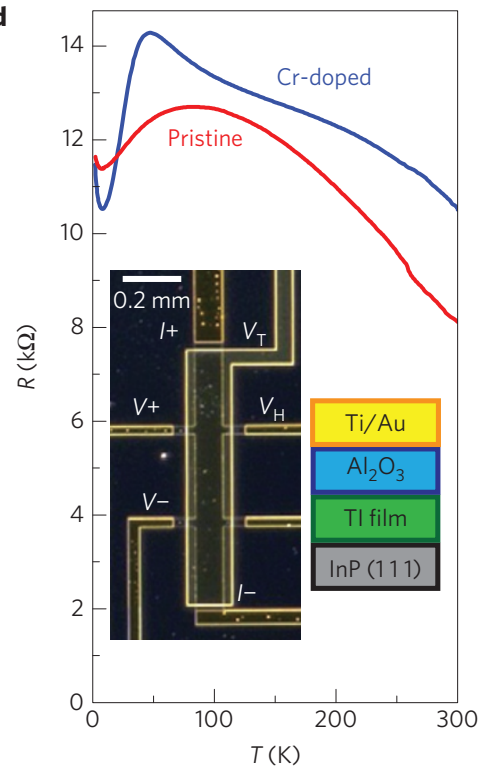

e

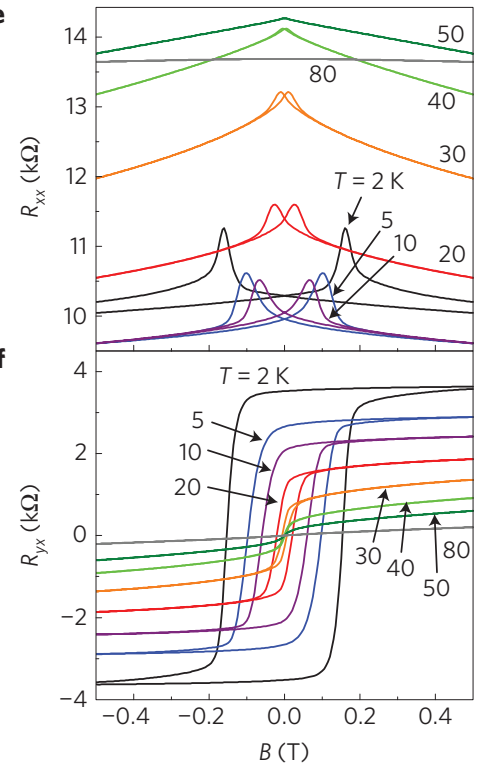

g

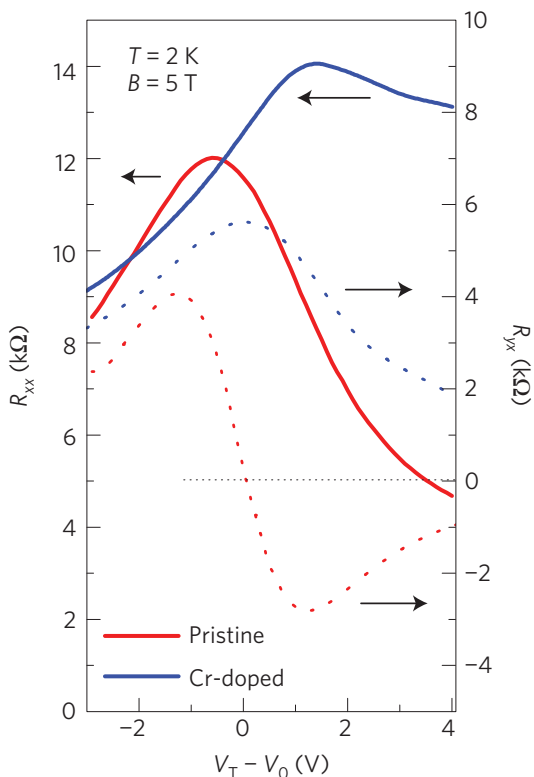

Figure 1 | Development of ferromagnetic topological insulator film devices. a, $\mathbf{b}, \mathrm{X}$-ray diffraction patterns for $\mathrm{Cr}_{x}\left(\mathrm{Bi}_{1-y} \mathrm{Sb}_{y}\right)_{2-x} \mathrm{Te}_{3}$ with $y=0.8$ for the pristine $(x=0)(\mathbf{a})$ and optimally magnetically doped $(x=0.22)(\mathbf{b})$ cases. $\mathbf{c}$, Detailed view of the $(00015)$ peak. The left and right insets show the atomic force microscope topography of $2 \mu \mathrm{m} \times 2 \mu \mathrm{m}$ areas of the pristine and doped films, respectively. $\mathbf{d}$, Resistance $R$ as a function of temperature $T$ for the films. The inset shows a lithographically defined device used in this study along with a schematic of the electrostatic gate structure. e,f, Longitudinal resistance $R_{x x}$ and transverse resistance $R_{y x}$, respectively, of the $\mathrm{Cr}$-doped film as a function of magnetic field $B$, showing hysteresis associated with the onset of ferromagnetic ordering near $T=40 \mathrm{~K}$. g, Gate voltage $V_{T}-V_{0}$ (relative to the zero-gap voltage) dependence of pristine and $\mathrm{Cr}$-doped films at $T=2 \mathrm{~K}$ and $B=5 \mathrm{~T}$, showing ambipolar behaviour.

in the bulk band gap of a TI such that, at high temperature, excitation of carriers into the bulk bands dominates transport, giving way to an intermediate temperature regime dominated by metallic surface conduction, and finally quantum corrections to conduction at the lowest temperatures ${ }^{25}$. On doping with $\mathrm{Cr}$, the most prominent difference observed is an enhancement in $R(T)$, peaking at a critical temperature $T_{\mathrm{C}}=45 \mathrm{~K}$. As shown in Fig. 1e,f, this $T_{\mathrm{C}}$ corresponds also to the onset of hysteresis in the longitudinal $R_{x x}(B)$ and transverse $R_{y x}(B)$ magnetotransport for the Cr-doped films, suggesting that $T_{\mathrm{C}}$ can be associated with the magnetic ordering temperature of the films. The behaviour in Fig. 1e,f is that of a typical ferromagnetic metal, where $R_{x x}$ has a peak at the coercive field $H_{\mathrm{C}}$ (here, $H_{\mathrm{C}}=0.16 \mathrm{~T}$ at $T=2 \mathrm{~K}$ ) and $R_{y x}$ is dominated by the anomalous Hall effect ${ }^{26}$. Above $T_{\mathrm{C}}$ we can estimate the electron mobility $\mu_{e}$ of the films; at $T=80 \mathrm{~K}$ we have $\mu_{e} \approx 270 \mathrm{~cm}^{2} \mathrm{~V}^{-1} \mathrm{~s}^{-1}$ (in comparable conditions the pristine films exhibit $\mu_{e}$ approaching $700 \mathrm{~cm}^{2} \mathrm{~V}^{-1} \mathrm{~s}^{-1}$ ). We note that the parameters $t$ and $x$ are optimized for this experiment by examining $R(T)$ and $R_{y x}(B)$ so as to retain metallic behaviour with the largest anomalous Hall response (Supplementary Methods).

To have in situ control of $\mu$, we patterned the films into Hall bars with deposited $\mathrm{Ti} / \mathrm{Au}$ top gates and $\mathrm{Al}_{2} \mathrm{O}_{3}$ gate dielectrics (Methods). An image of a patterned device is shown as the left inset of Fig. 1d, along with a schematic representation of the vertical structure in the right inset. For the films here, we are able to reach charge neutrality ( $\mu$ balanced between electron-like and hole-like) at a film-dependent top gate voltage $V_{\mathrm{T}}$, which we hereafter refer to as $V_{0}$. The results for both the pristine and doped films at $T=2 \mathrm{~K}$ and $B=5 \mathrm{~T}$ are shown in Fig. $1 \mathrm{~g}$ as a function of $V_{\mathrm{T}}$ relative to $V_{0}$. For the pristine film, we see ambipolar behaviour, indicated by the sign 


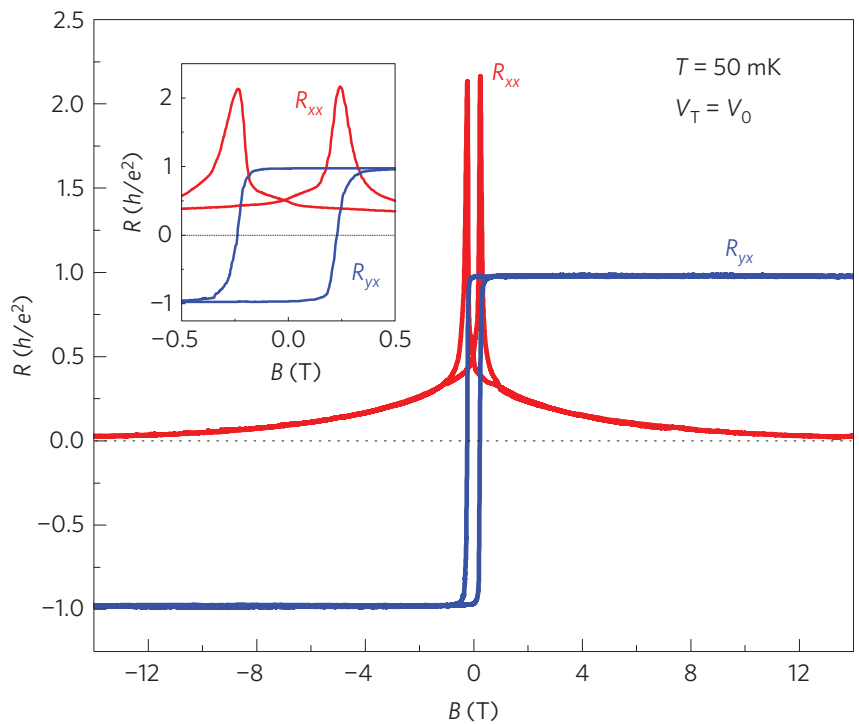

Figure 2 | Quantum anomalous Hall effect. Longitudinal resistance $R_{x x}$ and transverse resistance $R_{y x}$ of $\mathrm{Cr}$-doped film as a function of magnetic field $B$ at $T=50 \mathrm{mK}$ and with an optimally tuned gate voltage $V_{0} . R_{y x}$ exhibits a value of $0.98 \pm 0.003 \mathrm{~h} / \mathrm{e}^{2}$ whereas the longitudinal resistance falls with increasing $B$, reaching values $<0.03 \mathrm{~h} / \mathrm{e}^{2}$ at $B=14 \mathrm{~T}$. The inset shows the response at low magnetic field.

change in $R_{y x}\left(V_{\mathrm{T}}\right)$ at $V_{\mathrm{T}}=V_{0}$ and the simultaneous peak in $R_{x x}\left(V_{\mathrm{T}}\right)$; (ref. 25). For the doped film, we also observe a peak in $R_{x x}\left(V_{\mathrm{T}}\right)$, although it markedly less symmetric, along with a simultaneous variation in $R_{y x}\left(V_{\mathrm{T}}\right)$. Here, we define $V_{0}$ at the peak in $R_{y x}\left(V_{\mathrm{T}}\right)$, although no sign change occurs because of the large contribution of the anomalous Hall response. The observation of a maximum in the anomalous Hall response at charge neutrality is consistent with previously reported behaviour ${ }^{19}$.

Cooling to lower temperatures, we observe behaviour characteristic of the QAH effect. In Fig. 2, measurements of a doped film at $V_{\mathrm{T}}=V_{0}$ and $T=50 \mathrm{mK}$ are shown up to a magnetic field of $B=14$ T. $R_{y x}$ exhibits a value of $0.98 \pm 0.003 \mathrm{~h} / \mathrm{e}^{2}$, whereas the longitudinal resistance falls with increasing $B$, reaching values $<0.03 \mathrm{~h} / \mathrm{e}^{2}$ at $B=14 \mathrm{~T}$. The inset of Fig. 2 shows the response at low magnetic field, highlighting a remnant anomalous Hall resistance of $0.98 \mathrm{~h} / \mathrm{e}^{2}$. These results are similar to those previously reported ${ }^{8}$, although here a larger $B$ is required to suppress $R_{x x}\left(T_{\mathrm{C}}\right.$ and $H_{\mathrm{C}}$ are also different, see above). It seems that whereas a substantial $B$ is needed to support dissipation-less longitudinal transport, the Hall response is robust even in vanishing $B$, with the slight reduction from $h / e^{2}$ probably due to remnant conduction channels ${ }^{20}$.

To examine transport behaviour in the vicinity of the QAH state, we show the detailed evolution of $R_{x x}$ and $R_{y x}$ at $B=14 \mathrm{~T}$ at various temperatures as a function of $V_{\mathrm{T}}$ in Fig. 3a,b, respectively. A nearly dissipation-less $R_{x x}$ is observed over a narrow $V_{\mathrm{T}}$ range around $V_{0} \approx 3 \mathrm{~V}$ at $T=50 \mathrm{mK}$, which quickly weakens with increasing $T$ in a metallic fashion. There is a notable asymmetry for $R_{x x}$ on the electron and hole sides, but both sides become non-metallic at sufficiently large $\left|V_{\mathrm{T}}\right|$. For $R_{y x}$ we observe a plateau over a slightly larger $V_{\mathrm{T}}$ domain, which recedes with both increasing $T$ or for $V_{\mathrm{T}}$ significantly away from $V_{0}$. Mapping these curves into conductivities produces a systematic view of this evolution. In Fig. 3c-e we show parametric plots of $\left(\sigma_{x y}\left(V_{\mathrm{T}}\right), \sigma_{x x}\left(V_{\mathrm{T}}\right)\right)$, with $V_{\mathrm{T}}$ being the parameter, for various $B$ for $T=700 \mathrm{mK}, 200 \mathrm{mK}$, and $50 \mathrm{mK}$, respectively. At the lowest $T$ (Fig. 3e), the evolution from low $V_{\mathrm{T}}$ begins from large $\sigma_{x x}$ towards an apparent critical point at $\left(e^{2} / h, 0\right)$, although with a minor deviation consistent with $R_{y x}$ being slightly below $h / e^{2}$ and the finite remnant $R_{x x}$.
Interestingly, as $V_{\mathrm{T}}$ passes through $V_{0}$ (in the vicinity of the critical point) and further increases, curves at all $B$ collapse onto a single line nearly described by a semicircle of radius $e^{2} / 2 h$ centred at $\left(e^{2} / 2 h, 0\right)$, shown as a dashed line in Fig. $3 \mathrm{c}-\mathrm{e}$. This recalls the semicircular law derived to describe the transition between adjacent $\mathrm{QH}$ states or to the insulator ${ }^{27,28}$. The breakdown of this behaviour seems to be connected with the electron-hole asymmetry discussed above; we suggest that the asymmetry of the electronic structure with bulk valence band states being in closer proximity to the Dirac point may be the origin of this. As more readily expected from theory, the deviation from the semicircular behaviour increases with increasing $T$ (Fig. 3c,d). This is a preliminary connection of the present QAH state to known $\mathrm{QH}$ behaviour.

We can further probe the connection of the present system to $\mathrm{QH}$ states by examining the detailed temperature dependence of the conductivity tensor. In Fig. 4 a we show $R_{x x}(T)$ for several $V_{\mathrm{T}}$ for $B=0$ (after application of a large $B$ to saturate the magnetization $M)$. A crossover from non-metallic to metallic behaviour across $h / e^{2}$ is observed as $V_{\mathrm{T}}$ crosses $+4 \mathrm{~V}$. In Fig. $4 \mathrm{~b}$ we show $R_{y x}(T)$ measured under the same conditions, where we observe a change from increasing to decreasing $R_{y x}(T)$ with decreasing $T$ as $V_{\mathrm{T}}$ is increased across $+4 \mathrm{~V}$. This apparently complex crossover between two phases (one insulating and one metallic) is resolved by viewing these results from the perspective of the renormalization group (RG) properties of QH states.

As noted above, in the absence of a TRS-breaking magnetic field, $2 \mathrm{D}$ systems flow towards the insulating ground state as $T$ approaches zero (or equivalently as the system size $L$ diverges), a notion based on single parameter scaling analysis ${ }^{21}$. In the presence of broken TRS, however, the scaling function (the so-called $\beta$ function) involves two parameters. Theoretically, the behaviour of the conductivity tensor based on these $\beta$ functions under the condition of diverging $L$ under RG flow is suggested to be characterized by flow lines dictated by stable and unstable critical points in the parameter space of $\sigma_{x x}$ and $\sigma_{x y}$ (ref. 9). The law of corresponding states for $\mathrm{QH}$ systems, the rules that govern the symmetry relations under which $\mathrm{QH}$ states are identical and thus dictate the phase diagram of $\mathrm{QH}$ systems ${ }^{13}$, plays a key role in allowing us to quantitatively draw a comparison between experiment and this theoretical description.

The symmetries embodied by the law of corresponding states have been shown to impose a symmetry corresponding to the $\Gamma_{0}(2)$ modular subgroup on the associated conductivity tensor ${ }^{14}$. By writing the complex conductivity $\sigma=i \sigma_{x x}+\sigma_{x y}$, one can describe the behaviour of $\sigma$ in the upper half of the complex plane under RG flow. Here, we employ work motivated by the proposed duality of $\mathrm{QH}$ systems and $\mathcal{N}=2$ supersymmetric Yang-Mills theory rooted in their common $\Gamma_{0}(2)$ symmetry ${ }^{12}$. In analogy to RG flow studied in the latter ${ }^{29}$, it is postulated that the relevant $\beta$ functions are complex analytical functions of $\sigma$, from which the following function $f(x)$ can be derived

$$
f(x)=-\frac{\vartheta_{3}^{4}(x) \vartheta_{4}^{4}(x)}{\vartheta_{2}^{8}(x)}
$$

where $\vartheta_{i}(x)$ are Jacobi theta functions of the $i$ th kind, such that for $x=\mathrm{e}^{i \pi \sigma}$ equation (1) has a constant complex phase $\phi$ along RG flows-namely, $\arg f=\phi$ (ref. 12). We can then construct the RG flow diagram for the $\mathrm{QH}$ and insulating states by plotting the contours of $\arg f$ in $\sigma$. Several contours in the phase space covering the insulator and the $v=1$ filling QH state are shown in Fig. 4c, with arrows indicating the direction of flow with increasing length scale. In the vicinity of $(0,0)$ and $\left(e^{2} / h, 0\right)$ there are two stable fixed points (denoted by circles with a centre dot) corresponding to insulating and $\mathrm{QH}$ ground states, respectively. The unstable point (circle with 


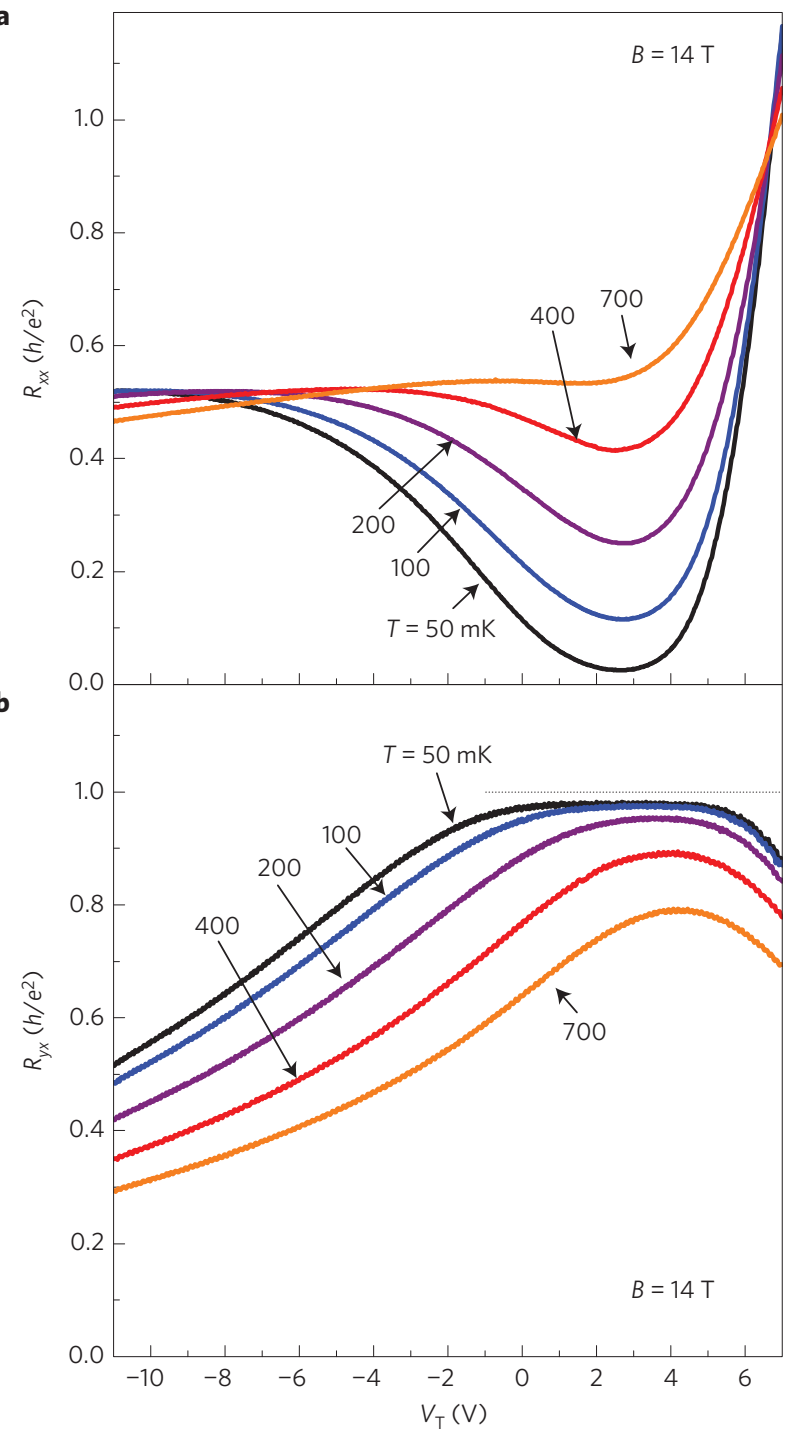

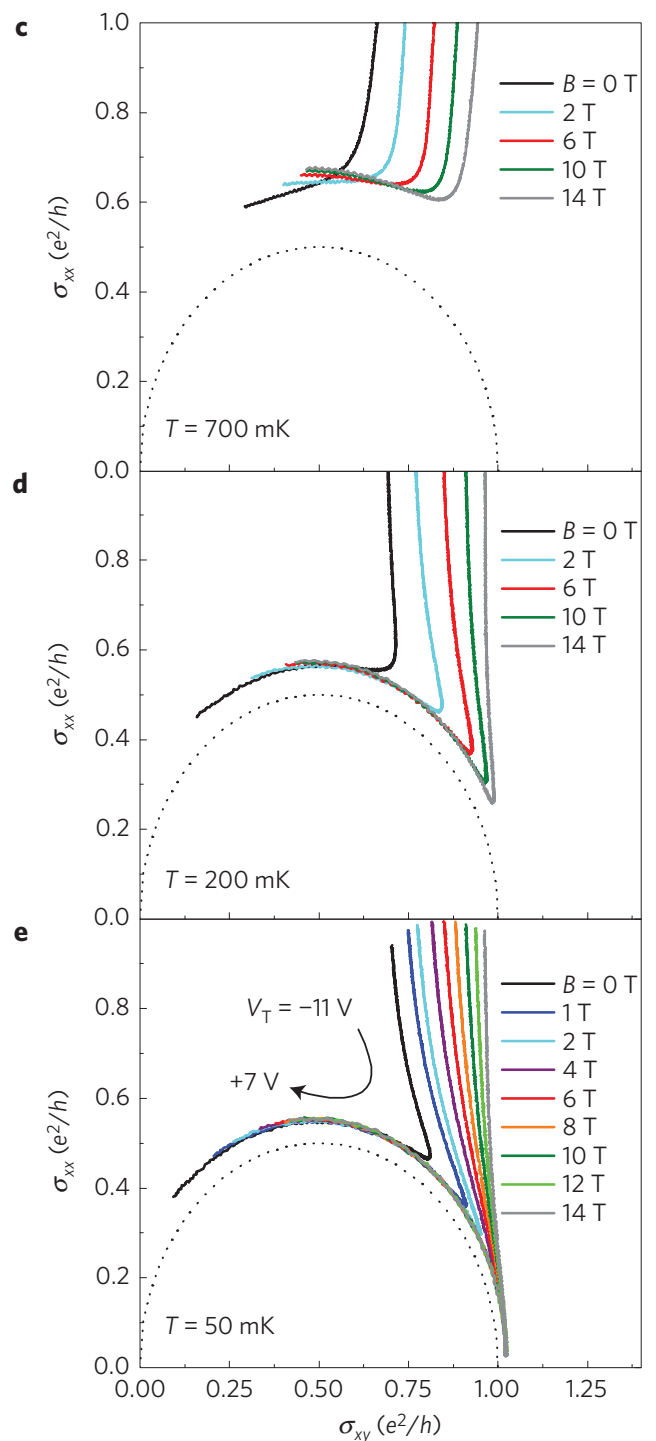

Figure 3 | Transport response near the quantum anomalous Hall regime. a, $V_{T}$ dependence of the longitudinal resistance $R_{x x}$ at $B=14 T$. $\mathbf{b}, V_{T}$ dependence of the transverse resistance $R_{y x}$ at $B=14 \mathrm{~T}$. c-e, Parametric plots of $\left(\sigma_{x y}\left(V_{T}\right), \sigma_{x x}\left(V_{T}\right)\right)$ for various values of $B$ at $T=700 \mathrm{mK}, 200 \mathrm{mK}$, and $50 \mathrm{mK}$, respectively. The direction of flow is clockwise for negative to positive gate voltage, as depicted by the arrow in $\mathbf{e}$. A semicircle of radius $\mathrm{e}^{2} / 2 \mathrm{~h}$ centred at $\left(e^{2} / 2 h, 0\right)$ is shown as a dashed line in each panel.

cross) indicates the transition regime or delocalization regime where extended states exist. These features reproduce the flows predicted by other methods ${ }^{9,11}$ and characterize the phase transitions of the $2 \mathrm{D}$ electron system with broken TRS. We note that setting $\phi=0$ recovers the condition for the semicircular law discussed above, which is not surprising as $\Gamma_{0}(2)$ symmetry serves as the basis for an alternate derivation ${ }^{30}$.

Turning to a comparison with our experimental results, we plot $\left(\sigma_{x y}\left(V_{\mathrm{T}}\right), \sigma_{x x}\left(V_{\mathrm{T}}\right)\right)$ for $B=0$ at various $V_{\mathrm{T}}$ in Fig. $4 \mathrm{~d}$. As $T$ is decreased (corresponding to increasing $L),\left(\sigma_{x y}\left(V_{\mathrm{T}}\right), \sigma_{x x}\left(V_{\mathrm{T}}\right)\right)$ seems to flow to one of two stable fixed points at $(0,0)$ and $\left(e^{2} / h, 0\right)$, with the pattern suggesting an unstable fixed point in the vicinity of $e^{2} / h(0.5,0.55)$. This unstable fixed point coincides closely with that expected for the delocalization transition of extended states producing universal singularities ${ }^{31}$. This behaviour thus qualitatively reproduces the characteristics of RG flow towards the insulating and $\mathrm{QH}$ ground states in Fig. 4c. Quantitatively, we find that equation (1) describes flow lines that capture our experimental result with reasonably high accuracy and precision, shown as the thin black curves in Fig. 4d. Previous experimental work has captured aspects of this flow in heterojunction $\mathrm{QH}$ systems ${ }^{10}$; this is clear evidence that the QAH state obeys the same symmetry rules. This flow is controllable with $V_{\mathrm{T}}$ even with $B=0$, suggesting that spontaneous $M$ in the QAH can drive identical behaviour to $B$ in $\mathrm{QH}$ systems (similar behaviour is observed in a field, see Supplementary Methods).

Comparing to previous systems in which RG flow has been less successful in the regime of classical percolation ${ }^{10}$, one conclusion here is that quantum localization plays a key role in determining the observed behaviour. The agreement between the behaviours of the QAH effect and QH effect in terms of RG flow may not be entirely surprising given that both the anomalous Hall effect and QH effect are described by field theories that are topological in origin ${ }^{11,26}$. This observation motivates a number of directions for future study. One extension of this analogy is to the larger global phase diagram of $\mathrm{QH}$ states for the QAH effect, which would predict that the $v=1$ QAH state would be connected continuously to other integer and fractional QAH states and that other exotic insulating states may be in close proximity ${ }^{13}$. If there are indeed neighbouring states this suggests that the corresponding phase transitions would belong to 

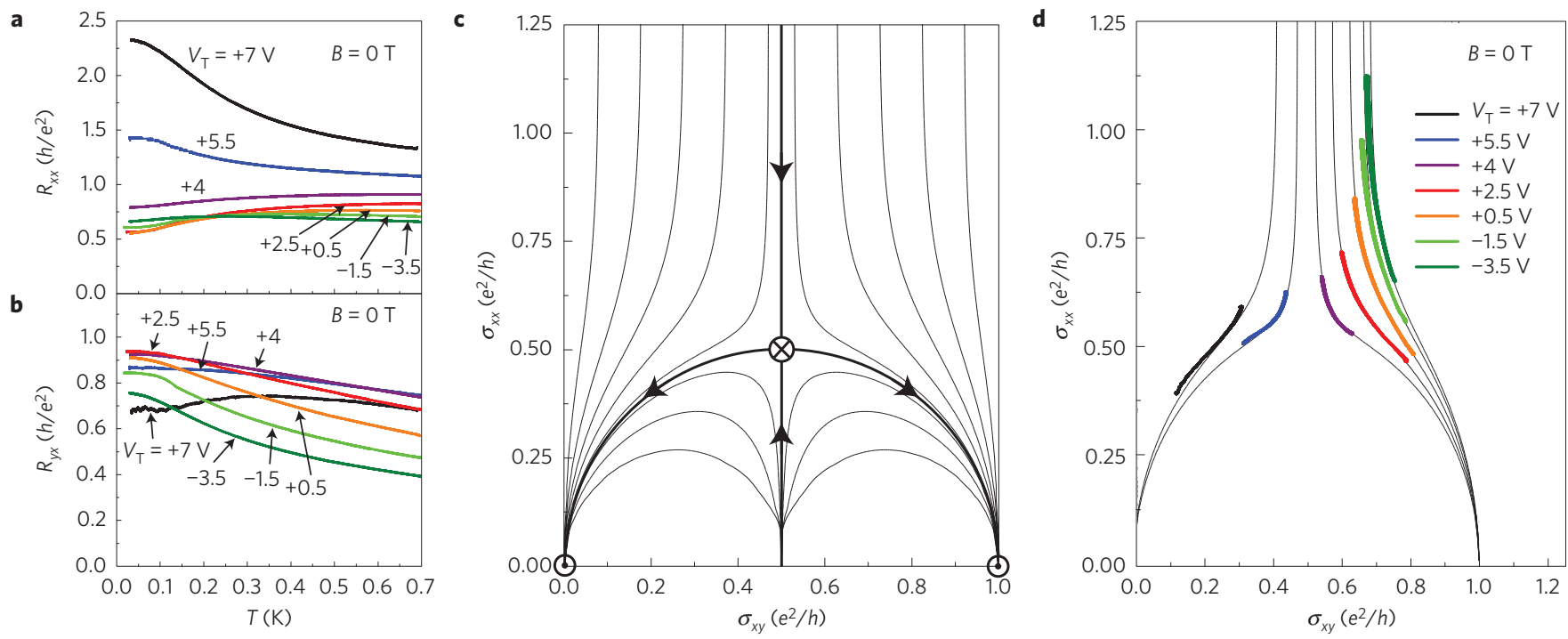

Figure 4 | Temperature driven renormalization group flow. a, Temperature dependence of the longitudinal resistance $R_{x x}$ at $B=0$ (after magnetic training) for various values of top gate voltage $V_{T}$. $\mathbf{b}$, Temperature dependence of the transverse resistance $R_{y x}$ at $B=0$ (after magnetic training) for various values of top gate voltage $V_{T}$. c, Calculation of renormalization group flow for a 2D electron system with insulating and quantum Hall stable points. $\mathbf{d}$, Flow in $\left(\sigma_{x y}\left(V_{T}\right), \sigma_{x x}\left(V_{T}\right)\right)$ observed at $B=0$ for various $V_{T}$. On decreasing $T$, the system evolves towards lower $\sigma_{x x}$. The thin lines are renormalization group flow lines calculated as discussed in the text.

the same universality class in the Anderson localization regime and would be of great interest for further study. As has been observed previously ${ }^{10}$, we would also predict this scaling would break down at elevated temperatures. More generally, experiments designed to detect the edge states and their properties associated with the QAH ground state would test our present understanding of this system ${ }^{32}$. We expect this framework for understanding the QAH effect will guide the discovery of new quantum states and phase transitions in the study of TIs and TRS breaking.

\section{Methods}

Thin films were grown by molecular beam epitaxy on epi-ready semi-insulating $\operatorname{InP}(111)$ at a substrate temperature of $260^{\circ} \mathrm{C}$ after annealing at $380^{\circ} \mathrm{C}$. The beam flux of Te relative to $\mathrm{Bi}, \mathrm{Sb}$, and $\mathrm{Cr}$ is kept at a ratio of of 20:1 to suppress $\mathrm{Te}$ vacancies. The elemental compositions of $\mathrm{Bi}, \mathrm{Sb}$ and $\mathrm{Cr}$ are estimated from the relative beam flux pressures checked before and after deposition. The growth rate of the films was approximately $0.2 \mathrm{QL} \mathrm{min}{ }^{-1}$. Transport measurements for $T>2 \mathrm{~K}$ are performed on unpatterned films with an excitation current of $1 \mu \mathrm{A}$ via electrical contacts made with $\mathrm{Au}$ wires and $\mathrm{Ag}$ paint in a commercial variable temperature cryostat. To correct for contact misalignment, the data in Fig. 1e,f are field symmetrized (unsymmetrized data is shown in Supplementary Fig. 7). For low-temperature measurements, films are capped ex situ after growth with $\mathrm{Al}_{2} \mathrm{O}_{3}$ deposited by atomic layer deposition (performed at $T<120^{\circ} \mathrm{C}$ to a thickness of $25 \mathrm{~nm}$ ) before subsequent processing by standard photolithography techniques and Ar ion milling (the etching is done with a brief exposure of less than 60 s). Electrical contacts and the top gate are made with e-beam evaporation of $\mathrm{Ti} / \mathrm{Au}$. Care is taken to use low $T<150^{\circ} \mathrm{C}$ processing to avoid degradation of the films. $R_{x x}$ and $R_{y x}$ reported here are the longitudinal (sheet) resistance and transverse (Hall) resistance, respectively. Measurements on devices are performed at low frequency $(1-3 \mathrm{~Hz})$ with lock-in amplifiers and voltage and current pre-amplifiers with an excitation current limited to $1 \mathrm{nA}$. We use a commercial dilution refrigerator equipped with a superconducting magnet. For device measurements we do not perform field symmetrization.

Received 25 January 2014; accepted 7 July 2014; published online 17 August 2014

\section{References}

1. Kane, C. L. \& Mele, E. J. Z2 topological order and the quantum spin Hall effect. Phys. Rev. Lett. 95, 146802 (2005).

2. Bernevig, B. A., Hughes, T. L. \& Zhang, S-C. Quantum spin Hall effect and topological phase transition in HgTe quantum wells. Science 314, 1757-1761 (2006).
3. Konig, M. et al. Quantum spin Hall insulator state in HgTe quantum wells. Science 318, 766-770 (2007).

4. Fu, L., Kane, C. L. \& Mele, E. J. Topological insulators in three dimensions. Phys. Rev. Lett. 98, 106803 (2007).

5. Hsieh, D. et al. A topological Dirac insulator in a quantum spin Hall phase. Nature 452, 970-974 (2008)

6. Yu, R. et al. Quantized anomalous Hall effect in magnetic topological insulators. Science 329, 61-64 (2010).

7. Nomura, K. \& Nagaosa, N. Surface-quantized anomalous Hall current and the magnetoelectric effect in magnetically disordered topological insulators. Phys. Rev. Lett. 106, 166802 (2011).

8. Chang, C-Z. et al. Experimental observation of the quantum anomalous Hall effect in a magnetic topological insulator. Science 340, 167-170 (2013).

9. Khmel'nitskii, D. E. Quantization of Hall conductivity. JETP Lett. 38, 552-556 (1983).

10. Wei, H. P., Tsui, D. C., Paalanen, M. A. \& Pruisken, A. M. M. Scaling of the Integral Quantum Hall Effect. Springer Series in Solid-State Sciences 71, 11-15 (1987).

11. Pruisken, A. M. M. Dilute instanton gas as the precursor to the integral quantum Hall effect. Phys. Rev. B 32, 2636-2639 (1985).

12. Dolan, B. P. Modular invariance, universality, and crossover in the quantum Hall effect. Nucl. Phys. B 554, 487-513 (1999).

13. Kivelson, S., Lee, D-H. \& Zhang, S-C. Global phase diagram in the quantum Hall effect. Phys. Rev. B 46, 2223-2238 (1992).

14. Lutken, A. A. \& Ross, G. G. Delocalization, duality, and scaling in the quantum Hall system. Phys. Rev. B 48, 2500-2514 (1993).

15. Liu, Q., Liu, C-X., Xu, C., Qi, X-L. \& Zhang, S-C. Magnetic impurities on the surface of a topological insulator. Phys. Rev. Lett. 102, 156603 (2009).

16. Abanin, D. A. \& Pesin, D. A. Ordering of magnetic impurities and tunable electronic properties of topological insulators. Phys. Rev. Lett. 106, $136802(2011)$.

17. Chen, Y. L. et al. Massive Dirac fermion on the surface of a magnetically doped topological insulator. Science 329, 659-662 (2010).

18. Wray, L. A. et al. A topological insulator surface under strong Coulomb, magnetic and disorder perturbations. Nature Phys. 7, 32-37 (2011).

19. Chang, C-Z. et al. Thin films of magnetically doped topological insulator with carrier-independent long-range ferromagnetic order. Adv. Mater. 25, 1065-1070 (2013).

20. Checkelsky, J. G., Ye, J., Onose, Y., Iwasa, Y. \& Tokura, Y. Dirac-fermion-mediated ferromagnetism in a topological insulator. Nature Phys. 8, 729-733 (2012).

21. Abrahams, E., Anderson, P. W., Licciardello, D. C. \& Ramakrishnan, T. V. Scaling theory of localization: Absence of quantum diffusion in two dimensions. Phys. Rev. Lett. 42, 673-676 (1979).

22. Levine, H., Libby, S. B. \& Pruisken, A. M. M. Electron delocalization by a magnetic field in two dimensions. Phys. Rev. Lett. 51, 1915-1918 (1983). 
23. Onoda, M. \& Nagaosa, N. Quantized anomalous Hall effect in two-dimensional ferromagnets: Quantum Hall effect in metals. Phys. Rev. Lett. 90, 206601 (2003)

24. Zhang, J. et al. Band structure engineering in $\left(\mathrm{Bi}_{1-x} \mathrm{Sb}_{x}\right)_{2} \mathrm{Te}_{3}$ ternary topological insulators. Nature Commun. 2, 574 (2011)

25. Checkelsky, J. G., Hor, Y. S., Cava, R. J. \& Ong, N. P. Bulk band gap and surface state conduction observed in voltage-tuned crystals of the topological insulator $\mathrm{Bi}_{2} \mathrm{Se}_{3}$. Phys. Rev. Lett. 106, 196801 (2011).

26. Nagaosa, N., Sinova, J., Onoda, S., MacDonald, A. H. \& Ong, N. P. Anomalous Hall effect. Rev. Mod. Phys. 82, 1539-1592 (2010).

27. Dykhne, A. M. \& Ruzin, I. M. Theory of the fractional quantum Hall effect The two-phase model. Phys. Rev. B 50, 2369-2379 (1994).

28. Hilke, M. et al. Semicircle: An exact relation in the integer and fractional quantum Hall effect. Europhys. Lett. 46, 775-779 (1999).

29. Seiberg, N. \& Witten, E. Electric-magnetic duality, monopole condensation, and confinement in $N=2$ supersymmetric Yang-Mills theory. Nucl. Phys. B 426, 19-52 (1994).

30. Burgess, C. P., Dib, R. \& Dolan, B. P. Derivation of the semicircle law from the law of corresponding states. Phys. Rev. B 62, 15359-15362 (2000).

31. Pruisken, A. M. M. Universal singularities in the integral quantum Hall effect. Phys. Rev. Lett. 61, 1297-1300 (1988).

32. Roth, A. et al. Nonlocal transport in the quantum spin Hall state. Science 325, 294-297 (2009)

\section{Acknowledgements}

We are grateful to N. Nagaosa, B.-J. Yang and A. F. Young for fruitful discussions, and M. Nakano, T. Hatano, S. Shimizu, M. Kubota and S. Ono for technical support. This research is supported by the Japan Society for the Promotion of Science (JSPS) through the 'Funding Program for World Leading Innovative R\&D on Science and Technology (FIRST Program),' initiated by the Council for Science and Technology Policy (CSTP), and by JPSP Grant-in-Aid for Scientific Research, Nos. 24224009, 24226002 and 25871133. This work was carried out by joint research of the Cryogenic Research Center, the University of Tokyo.

\section{Author contributions}

J.G.C. and R.Y. grew and characterized the films. J.G.C., Y.K. and J.F. performed the low-temperature measurements. J.G.C. analysed the data and wrote the paper with contributions from all authors. A.T., K.S.T., M.K. and Y.T. contributed to discussion of the results and guided the project. Y.T. conceived and coordinated the project.

\section{Additional information}

Supplementary information is available in the online version of the paper. Reprints and permissions information is available online at www.nature.com/reprints.

Correspondence and requests for materials should be addressed to J.G.C.

\section{Competing financial interests}

The authors declare no competing financial interests. 